\title{
Pharmacoeconomic evaluation of treatment effectiveness with selected biologic treatment in rheumatoid arthritis therapy
}

\author{
Krzysztof Kowalik ${ }^{1,2}$, Małgorzata Węgierska ${ }^{2,3}$, Tacjana Barczyńska ${ }^{2,3}$, Sławomir Jeka ${ }^{2,3}$ \\ ${ }^{1}$ Non-public Health Care Centre „Nasz Lekarz”, Bydgoszcz, Poland \\ 2Department of Rheumatology and Connective Tissue Diseases, Jan Biziel University Hospital No. 2 in Bydgoszcz, Poland \\ ${ }^{3}$ Ludwik Rydygier Collegium Medicum in Bydgoszcz, Nicolaus Copernicus University in Toruń, Poland
}

\begin{abstract}
Objectives: Modern treatment of autoimmune diseases is becoming increasingly widely used. We owe it to the continuous and rapid development of biotechnology, molecular biology, immunology, and biochemistry. The proven effectiveness of biological therapy in rheumatoid arthritis (RA) should result in its widespread use. At present, only about $1 \%$ of patients with RA have access to biological therapy in Poland.

Material and methods: The study material was retrospectively collected in the Rheumatology and Systemic Tissue Diseases Clinic and Rheumatology Outpatient Clinic in dr Jan Biziel University Hospital No. 2 in Bydgoszcz 2009-2014. Patients were divided into 3 groups: patient receiving infliximab, etanercept and adalimumab.

Results: The study involved analyses of cost effectiveness. The time horizon of patient documentation analysis ranged from the time a patient was enrolled to infliximab, etanercept or adalimumab therapy until remission of the disease. The majority of patients achieved remission in the case of adalimumab treatment (85.29\%), followed by etanercept (74.07\%), then infliximab (37.21\%). Taking into account the DAS28 parameter, analysis was performed using medical costs of the analyzed treatment regimens. For this purpose, the incremental cost-effectiveness ratio (ICER) was calculated. According to the analysis, obtaining one DAS28 unit, replacing infliximab with etanercept, would cost PLN 4096467 . Higher costs would be required in the case of replacement of infliximab with adalimumab - PLN 43076 08. Obtaining one additional DAS28 unit (in this case, a decrease in DAS28 by one unit) by introducing adalimumab instead of etanercept would amount to PLN 4540974.

Conclusions: Undoubtedly, the pharmacoeconomic analysis makes it easier to decide on the appropriate treatment. Therefore, its implementation should be a widely used solution not only for RA, but also for other diseases. Health care and other entities' systems should also be improved in such a way that the data needed for pharmacoeconomic analysis are fully available.
\end{abstract}

Key words: rheumatology, rheumatoid arthritis, effectiveness of treatment of rheumatoid arthritis, pharmacoeconomic evaluation, biological treatment.

\section{Introduction}

Modern therapeutic treatment of autoimmune diseases is becoming increasingly widely used. We owe it to the continuous and rapid development of biotechnology, molecular biology, immunology, and biochemistry. The introduction of biological therapy in rheumatoid arthritis (RA) was a breakthrough in the standard approach to the treatment of this disease. Although the RA pathogenesis is still not fully understood, intercellular mechanisms, the role of individual cells, and proinflammatory

Address for correspondence:

Krzysztof Kowalik, Department of Rheumatology and Connective Tissue Diseases, Jan Biziel University Hospital No. 2 in Bydgoszcz,

75 Ujejskiego St., 85-168 Bydgoszcz, Poland, e-mail: kpmk@vp.pl

Submitted: 26.04.2018; Accepted: 9.08.2018 
cytokines, responsible for the persistence of high disease activity, have been identified. The introduction of medications from the group of TNF- $\alpha$ inhibitors turned out to be a proverbial milestone. These are low molecular weight proteins produced by genetic engineering, which, among other properties, have anti-inflammatory effects on the receptors, proinflammatory cytokines, and inflammatory cells. In most patients, their use in the treatment of RA results in a rapid therapeutic effect in the form of lowering disease activity and improving functional status. The causal treatment of RA is not currently available, but the recommended solution is therapy individualization and a systematic health assessment, preceded by an early and correct diagnosis.

Scientific evidence of biological therapy in RA should result in its widespread use. At present, only about 1\% of patients with RA have access to biological therapy in Poland [1-3]. We are far behind leading countries such as Sweden, Germany or England, where the level is over $20 \%$ [2]. The paper evaluates the effectiveness of biological therapy with three selected medications: infliximab, etanercept, and adalimumab.

\section{Material and methods}

The study material was retrospectively collected in the Rheumatology and Systemic Tissue Diseases Clinic and Rheumatology Outpatient Clinic in dr Jan Biziel University Hospital No. 2 in Bydgoszcz, CM, Nicolaus Copernicus University in Torun, in the years of 2009-2014. Data are based on the records of patients with rheumatoid arthritis diagnosed according to the 1987 ACR criteria, who were treated with etanercept, infliximab, and adalimumab. For the analysis, 104 patients were enrolled.

Patients were divided into 3 groups:

1. Patients receiving infliximab (INF) were a group of 43 persons (30 women and 13 men) aged 27 to 71 years (average age was 47 years). At the time of initiation of biological therapy, men had RA for an average of 6.5 years, women for 9 years.

2. Patients receiving etanercept (ETA) were a group of 27 persons ( 24 women and 3 men) aged 20 to 60 years (average age was 51 years). At the time of initiation of biological therapy, men had RA for an average of 13 years, women for 12 years.

3. Patients receiving adalimumab (ADA) were a group of 34 persons ( 23 women and 11 men) aged 25 to 77 years (average age was 54 years). At the time of initiation of biological therapy, men had RA for an average of 4.5 years, women for 8 years.

Patients have received medications under the $\mathrm{Na}$ tional Health Fund (Narodowy Fundusz Zdrowia, NFZ) therapeutic programs. Each patient included in the medication program had to meet appropriate criteria for inclusion and exclusion set by the National Health Fund.

Patients treated with infliximab received doses ranging from $150 \mathrm{mg}$ to $300 \mathrm{mg}$. Patients took infliximab in weeks 0, 2, 6 and then every 8 weeks. The medication was used in combination with methotrexate (administered at doses of $15 \mathrm{mg}$ to $25 \mathrm{mg}$ once a week). Infliximab was administered as an intravenous infusion. Each infliximab vial, containing $100 \mathrm{mg}$ of the product, was before infusion dissolved in $10 \mathrm{ml}$ of water for injections and then an appropriate content was transferred to a $0.9 \%$ sodium chloride solution of $250 \mathrm{ml}$ volume. On the day of administration, patients were admitted to the clinic in the morning. Prior to each administration, laboratory tests were performed and the number of painful and swollen joints and the subjective assessment of pain and severity of patient disease activity were determined. Following patient observation for potential infusion-related side effects, the patient left the hospital. In patients with no improvement or no response to the treatment with infliximab, the treatment was changed to another TNF- $\alpha$ inhibitor: etanercept or adalimumab, or to rituximab, according to National Health Fund guidelines.

Patients treated with etanercept received it subcutaneously at a dose of $50 \mathrm{mg}$ weekly. The medication was administered in combination with methotrexate (administered at doses of $15 \mathrm{mg}$ to $25 \mathrm{mg}$ once a week). Patients were referred for monthly check-ups to the Rheumatology Outpatient Clinic.

Patients treated with adalimumab received it subcutaneously at the dose of $40 \mathrm{mg}$ every two weeks. The medication was administered in combination with methotrexate (administered at doses of $15 \mathrm{mg}$ to $25 \mathrm{mg}$ once a week), or as monotherapy. Patients were referred for monthly check-ups to the Rheumatology Outpatient Clinic.

The study uses analyses of cost effectiveness. The study was conducted in accordance with the Good Pharmacoeconomic Practice guidelines. The effectiveness of RA therapy was compared using three alternative biological medications: infliximab, etanercept, and adalimumab. The time horizon of patient documentation analysis ranged from the time a patient was enrolled to infliximab, etanercept or adalimumab therapy until remission of the disease. For patients who did not achieve remission, 24 months were considered for the follow-up period.

The effectiveness of the treatment was determined on the basis of the Disease Activity Score and the following parameters:

- Disease Activity Score 28 (DAS28),

- disease activity determined by the doctor using a visual analogue scale (VAS), 


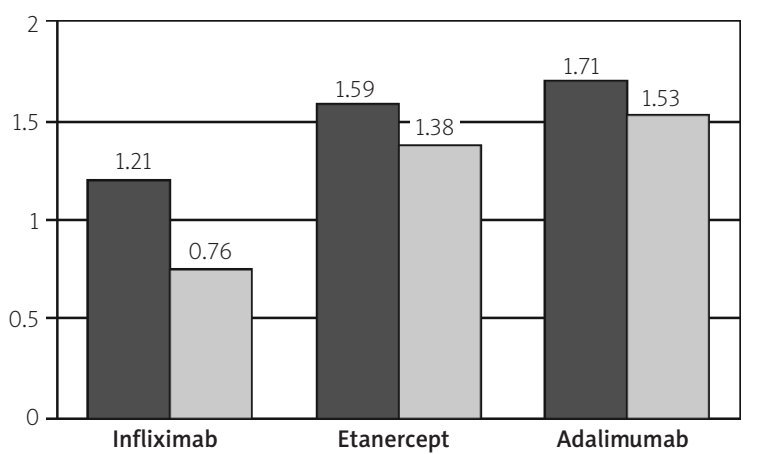

Fig. 1. Summary of average HAQ-DI values according to treatment regimen.

Table I. Summary of QALY values according to treatment regimen

\begin{tabular}{|cccc|}
\hline & Infliximab & Etanercept & Adalimumab \\
\hline QALY & 1.71 & 0.74 & 0.60 \\
\hline
\end{tabular}

- disease activity determined by the patient using a VAS,

- erythrocyte sedimentation rate (ESR),

- C-reactive protein concentration (CRP).

The quality of life was assessed on the basis of the completed questionnaire HAQ-DI. The patient had the opportunity to mark the answers connected with daily activities in 8 domains: dressing up and washing, morning waking, eating, walking, personal hygiene, lifting, grasping, other activities. The answer was scored on a scale from 0 to 3, where 0 meant performing the activity without any difficulty, 1 - with little difficulty, 2 - with great difficulty, 3 - impossible to perform. The result is the sum of the highest score in each domain, divided by the number of domains.

It was also decided to calculate the QALY (quality-adjusted life years) parameter, using changes in HAQ values over time, for individual therapies. This was done using statistics and by calculating the area under the curve (AUC), to give the QALY value.

\section{Results}

There was a significant decrease in VAS values in all groups compared. No improvement was observed in only two patients treated with adalimumab. Also the mean values of VAS according to physicians did not differ significantly from the values indicated by patients.

The majority of investigated patients achieved remission in the case of adalimumab treatment $(85.29 \%$; 29 of 34 patients), followed by etanercept (74.07\%; 20 of 27 patients), and the fewest patients achieved remission in the case of infliximab (37.21\%; 16 of 43 patients). In all studied treatment regimens, the average HAQ-DI value was lowered. The greatest difference was in patients treated with infliximab (0.45). According to patients who received etanercept, their health improved by an average of 0.21 (Fig. 1).

Evaluation of patients receiving adalimumab differed from the initial one by 0.18. During the two-year follow-up period, the best QALY results were obtained for infliximab treatment. Patients receiving etanercept and adalimumab rated their quality of life to be worse (Table I).

While treating patients in a therapeutic program, DAS28, CRP level, and ESR were used to assess disease activity. Average values per patient are shown in Table II.

According to the EULAR criteria for DAS28, the majority of patients achieved remission or low disease activity. The highest percentage of DAS $28 \leq 2.6$ was obtained with adalimumab treatment - up to $85 \%$. $74 \%$ of etanercept-treated patients and $37 \%$ of infliximab-treated patients also achieved remission. Low disease activity was reported in 17 patients (40\%) treated with infliximab, 4 (15\%) treated with etanercept, and 3 (9\%) treated with adalimumab. Moderate activity was observed in $23 \%$ of patients treated with infliximab, $11 \%$ treated with etanercept, and 6\% treated with adalimumab (Fig. 2).

Table III shows the values that were used to perform the incremental analysis in the form of the incremental cost-effectiveness ratio.

As treatment with infliximab, according to the obtained results, is a cheaper and more effective method

Table II. Average values of Disease Activity Score according to treatment regimen

\begin{tabular}{|lccccccccc|}
\hline & \multicolumn{3}{c}{ Infliximab } & \multicolumn{3}{c}{ Etanercept } & \multicolumn{3}{c|}{ Adalimumab } \\
\hline Parameter & DAS28 & $\begin{array}{c}\text { ESR } \\
(\mathrm{mm} / \mathrm{h})\end{array}$ & $\begin{array}{c}\text { CRP } \\
(\mathrm{mg} / \mathrm{l})\end{array}$ & DAS28 & $\begin{array}{c}\text { ESR } \\
(\mathrm{mm} / \mathrm{h})\end{array}$ & $\begin{array}{c}\text { CRP } \\
(\mathrm{mg} / \mathrm{l})\end{array}$ & DAS28 & $\begin{array}{c}\text { ESR } \\
(\mathrm{mm} / \mathrm{h})\end{array}$ & $\begin{array}{c}\text { CRP } \\
(\mathrm{mg} / \mathrm{l})\end{array}$ \\
\hline Initial & 6.12 & 32.21 & 29.42 & 6.04 & 35.52 & 18.99 & 6.05 & 30.68 & 14.94 \\
\hline Final & 2.82 & 17.21 & 7.70 & 2.53 & 17.56 & 6.67 & 2.35 & 16.99 & 5.50 \\
\hline Decrease & 3.30 & 15.00 & 21.72 & 3.51 & 17.96 & 12.32 & 3.70 & 13.69 & 9.44 \\
\hline Percentage (\%) & 53.92 & 46.57 & 73.83 & 58.11 & 50.56 & 64.88 & 61.16 & 44.62 & 63.19 \\
\hline
\end{tabular}

CRP - C-reactive protein; DAS28 - Disease Activity Score 28; ESR - erythrocyte sedimentation rate 
(based on the HAQ-DI and QALY), there is no need for an effectiveness analysis. However, taking into account the DAS28 parameter, such analysis was performed using medical costs of the analyzed treatment regimens. For this purpose, the incremental cost-effectiveness ratio (ICER) was calculated. According to the analysis, obtaining one DAS28 unit, replacing infliximab with etanercept, would cost PLN 40964 67. Higher costs would be required in the case of replacement of infliximab with adalimumab - PLN 43076 08. Obtaining one additional DAS28 unit by introducing adalimumab instead of etanercept would amount to PLN 4540974.

\section{Discussion}

In Poland, about 1\% of RA patients have access to upto-date treatment [1]. Access to biological therapy for RA patients in neighboring countries with similar demographic structure is more than 1\% [2]. Among highly developed countries in Western Europe, the percentage of patients treated with biologic medications is up to $20 \%$ (as is the case in Sweden, England, and Germany) (Fig. 3) [3].

The analysis was performed to compare the effectiveness of the treatment from the perspective of the doctor and the patient. It involved 104 patients. According to the subjective assessment of the VAS, both according to the patient and the doctor, the greatest difference between the initial and the final value was obtained for etanercept, followed by infliximab, and adalimumab. It should be noted that in all compared groups, a significant decrease in VAS value was noted. The second parameter, which reflects the patient's well-being and functional capacity, was the HAQ questionnaire. The greatest difference was observed in the patients treated with infliximab. It was more than two times higher than in the case of patients receiving etanercept and adalimumab. The assessment of disease activity from a doctor's perspective was presented by means of indicators such as DAS28, CRP, or ESR. Among the 104 patients, most achieved remission or low disease activity as defined by the EULAR criteria. The largest proportion of patients with remission was in the group treated with adalimumab (85.29\%), followed by etanercept (74.07\%), and infliximab (37.21\%).

The study also uses the QALY parameter, which provides usability as a measure of the benefits of the procedure - mortality and morbidity - that is, it is the combination of quantitative and qualitative gains. However, there are doubts about the appropriateness of QALY as a universal benefit measure. Attention was drawn to the fact that QALY, as an expression of life years adjusted for quality, takes into account both the length of life and the quality of the experience, but ignores the distribution of the determinant of health in society [4]. A better method
Table III. Difference in HAQ-DI, QALY, and improvement of DAS28 and direct costs according to treatment regimen per patient

\begin{tabular}{|lccc|}
\hline & Infliximab & Etanercept & Adalimumab \\
\hline $\begin{array}{l}\text { Decrease } \\
\text { of HAQ-DI }\end{array}$ & 0.45 & 0.21 & 0.18 \\
\hline QALY & 1.71 & 0.74 & 0.60 \\
\hline $\begin{array}{l}\text { Improvement } \\
\text { of DAS28 }\end{array}$ & 3.30 & 3.51 & 3.70 \\
\hline $\begin{array}{l}\text { Average medical } \\
\text { cost per patient }\end{array}$ & $64,263.61$ & $72,866.19$ & $81,494.04$ \\
\hline
\end{tabular}

DAS28 - Disease Activity Score 28; HAQ-DI - Health Assessment Questionnaire Disability Index; $Q A L Y$ - quality-adjusted life years

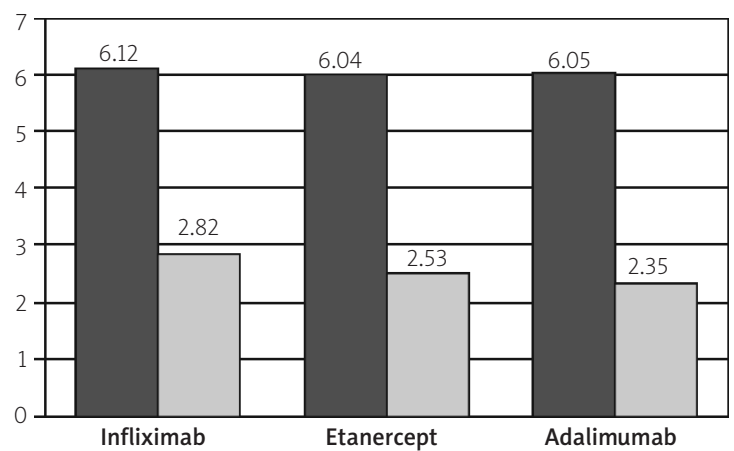

Fig. 2. Average DAS28 values according to treatment regimen.

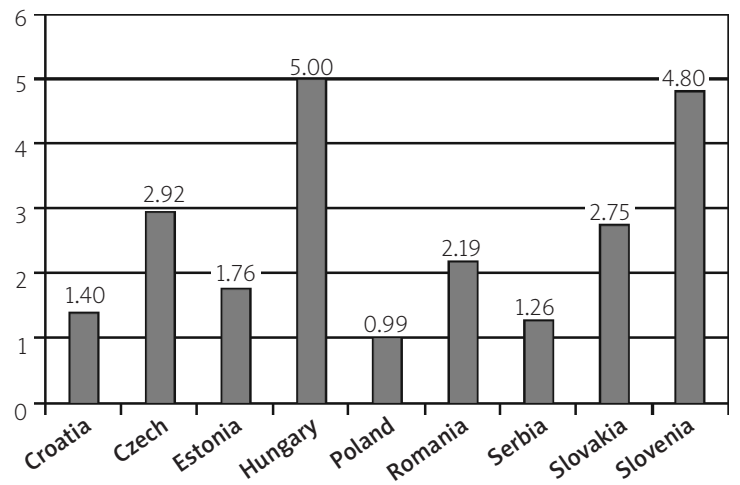

Fig. 3. Comparison of the number of patients (\%) with RA on biological treatment in different European countries [3].

has not been developed yet, and QALY is still a widely used measure in pharmacoeconomic analysis, especially in terms of cost-effectiveness analysis.

An analysis was performed using the incremental cost-effectiveness ratio (ICER). It made it possible to 
compare the cost-effectiveness of different preparations. By using this analysis, it is possible to estimate how much more a treatment may additionally cost or how much could be saved by replacing the current procedure with an alternative procedure.

It has already been demonstrated that the action of anti-TNF- $\alpha$ biological medications is much more effective when combined with disease-modifying antirheumatic drugs (DMARD) [5-7]. These therapies have produced very good clinical effects that have been observed throughout the world and in this study.

In the ATTRACT study [8] with low doses of MTX in combination with infliximab, approximately $60 \%$ of patients achieved an ACR20 response in 8-12 weeks. Patients had decreased inflammation, improved functional status and quality of life, and reduced destruction of joints in the radiological image. This study also compared MTX/placebo treatment to infliximab combined with MTX in patients who showed an inadequate response to MTX treatment. After one year of therapy, patients receiving infliximab and MTX showed significantly greater improvement in ACR20, ACR50, and ACR70 than patients treated with MTX monotherapy. The multicenter BeST study (Behandel Strategieën) [9] confirmed that the administration of infliximab in the early phase of RA was associated with a significant reduction in disease progression, progression of radiological changes, and remission of the disease that persisted even after discontinuation of treatment. The study also shows that infliximab may induce the formation of new T-cell subclasses that may renew the synovial membrane immune tolerance [9].

The effectiveness of adalimumab has been demonstrated in many clinical trials. One of the main ones, deserving of attention, is the ARMADA phase III study comparing the effects of adalimumab and MTX therapy with placebo and MTX. Almost $69 \%$ of patients achieved an ACR20 response, and in many patients it was found in radiological images that the progression of joint destruction had stopped. The good clinical response and effectiveness were maintained during the 4-year open-label extension study, even with reduced corticosteroid treatment and lowering the MTX dose [10]. Adalimumab has also been tested in patients with early RA. A better response to ACR 20, 50 and 70 was obtained, joint destruction in radiology was decreased, and quality of life and functional status were improved [10].

Patients treated with $25 \mathrm{mg}$ of etanercept had a response at ACR20, ACR50, and ACR70 levels of $62 \%, 41 \%$, and $15 \%$, respectively. This study contributed to the registration of etanercept in the indication for RA and confirmed the effectiveness of the therapy after failure of DMARD treatment [11]. It has also been proven that etanercept in combination with MTX produces better results than MTX or etanercept monotherapy. An example is the TEMPO study, where a statistically significant difference in clinical parameters was achieved, that is, the achievement of ACR20, ACR50, and ACR70 responses, HAQ, and DAS level [11]. This study also compared the effect of treatment on the progression of radiological changes in hands and feet. In the majority of patients (approximately 80\%), in the case of combined treatment (etanercept $+\mathrm{MTX}$ ), no radiographic progression was observed after one year [11].

The results of studies conducted on adalimumab, etanercept, and infliximab have been collected and compared [12]. It appears that the most effective treatment was achieved with adalimumab, followed by etanercept, and infliximab. This is evidenced by the percentage of responses ACR20, 50, and 70. For comparable treatments, the results are similar to the analysis performed in our own study. However, the study uses disease activity expressed by DAS28 as an evaluation of treatment effectiveness. Evaluation of treatment activity by ACR improvement criteria and EULAR response criteria (based on DAS28) may differ. It should be noted that the ACR criteria are a dichotomous measure, showing improvement or lack of it. The EULAR criteria allow for the classification of patients in terms of response to treatment, which in the case of the analysis in this paper turned out to be a better choice.

The assessment of the quality of life and the physical activity of the patient was performed using the HAQ questionnaire. In our study, it was found that the largest difference in the HAQ level between the initiation and end of treatment was achieved for infliximab, then etanercept, and adalimumab. The improvement resulting from the difference in $\mathrm{HAQ}$ values can be observed in almost every trial that has tested the effectiveness of biological therapy. However, the results of these studies are different and depend on many factors. The obtained HAQ difference value for infliximab in our study differs from the values reported in other studies - 0.45 vs. $0.10,0.6$ or 0.22 [13-15]. Differences can also be noted by comparing the results for adalimumab (0.18 vs. 0.08 and 0.34 ) or etanercept (0.21 vs. 0.12 or 0.36$)$ [13]. It is true that the initial improvement (significant reduction in the HAQ result), resulting from initiation of biological therapy, is lost over time. The increase in the HAQ value is the greater, the faster the biological treatment is stopped. However, this observation does not apply to every patient. Sometimes remission lasts for a considerable period of time. Nevertheless, it was found that after discontinuation of biological therapy, the average $\mathrm{HAQ}$ result increased by 0.125 [15]. In the discussion, the main source of comparisons was foreign articles. Research ar- 
ticles that focus on the population in Poland have not been found. Interpreting the HAQ results, also the stage of the disease should be taken into account, as both the effects of the disease process and its features affect physical disability. Nevertheless, it should be noted that regardless of the population and stage of the disease, the mean difference in $\mathrm{HAQ}$ values indicates that from the perspective of the patient the improvement has been achieved.

Taking into account the quality of life that is fundamentally affected by the treatment, it is important to consider a parameter that best describes and summarizes the results. In the paper, QALY was used as a measure of the outcome of a health program that combines the quality of life evaluation at the time when patients were treated. QALY was calculated based on HAQ-DI results. Unfortunately, the National Health Fund health programs do not provide patients with the most common quality of life questionnaire used for QALY calculation, EQ-5D. Therefore, the results obtained cannot be directly compared with others. It is also relevant that the duration of patient observation lasted up to 2 years. For this reason, not expected lifetime, but the average value of months for a given therapy was used to calculate QALY. Therefore, an indirect analysis was performed, comparing the results of QALY with the results of other scientific studies.

The available results are not always unambiguous. Although, when analyzing the results of treatment with infliximab, etanercept, and adalimumab, we get similar QALY values, the order of these results is very different. Authors of the publications present different research results and different methods. Kaczor and Wójcik in their study [16], using the Markov model, obtained QALY for infliximab 6.720, etanercept 7.080, and for adalimumab 7.153. Wailoo et al. [17] developed a model that recalculated the HAQ-DI results to the adjusted years for the quality of life. Their results were: infliximab -7.64 , etanercept - 7.66, and adalimumab-7.64. In one of the latest publications, Hidalgo-Vega [18] also benefited from the Markov model, obtaining QALY for infliximab 6.318, etanercept 6.462, and for adalimumab 6.430 . Chen et al. [7] compiled a comprehensive report on the cost-effectiveness analysis of biological medications, especially paying attention to treatment with infliximab, etanercept, and adalimumab. Their model assumed the use of Quality of Life (QoL) results obtained directly or through the conversion of HAQ-DI values. For this purpose, they used the Birmingham Rheumatoid Arthritis Model (BRAM), which allowed for a number of variations depending on the improvement of the HAQ result, mortality, or effectiveness of treatment. In every possible compilation, QALY results were ranked in the order (from the highest to the lowest): etanercept, adalimumab, infliximab. It should be emphasized that the results for adalimumab and infliximab were similar (differences in the range 0.02-0.04) [7].

The available results seem to be slightly different from the results obtained in this study. The obtained QALY values fall into a much smaller numerical range. However, this is due to the statistical method used and the observation time. When considering results in terms of the order, we also notice the difference. QALY values obtained in our study show the most beneficial therapy to be infliximab (1.71), followed by etanercept (0.74), and adalimumab (0.60). In the cited scientific works, etanercept and adalimumab are best in this respect. It should be noted, however, that discrepancies in QALY values compared to infliximab are generally in the range of hundredths. An important argument is that the data on infliximab contained information from patients who, after failure of infliximab treatment, were switched to adalimumab, etanercept, or rituximab therapy. Nevertheless, the QALY result for infliximab in our study is the most favorable. Currently, the study is focusing on the comparison of economically advantageous medications, that is, biosimilar medications. In a population demographically similar to the Polish one - the Bulgarian population - it was concluded that a medication biosimilar to infliximab (5.42) had better QALY results than adalimumab treatment (5.35) [19]. The observation itself covered a period of 10 years.

\section{Conclusions}

Undoubtedly, the pharmacoeconomic analysis makes it easier to decide on the appropriate treatment. Therefore, its implementation should be a widely used solution not only for RA, but also for other diseases. Health care and other entities' systems should also be improved in such a way that the data needed for pharmacoeconomic analysis are fully available. Existing restrictions impede easy access to the data about full direct and indirect costs. This is especially important because the economic balance is an essential element of complete pharmacoeconomic analysis.

\section{The authors declare no conflict of interest.}

\section{References}

1. Kobelt G, Kasteng F. Access to innovative treatments in rheumatoid arthritis in Europe. A report prepared for the European Federation of Pharmaceutical Industry Associations (EFPIA) 2009: 1-92.

2. Orlewska E, Wiland P. Access to biologic treatment for rheumatoid arthritis in Central and Eastern European (CEE) countries. Med Sci Monit 2011; 17: 1-13. 
3. Stajszczyk M. Raport. Biological treatment in rheumatic diseases in Poland in 2013: 11.

4. Johannesson M, Gerdtham UG. A note on the estimation of the quality - efficiency trade-off for QALYs. J Health Econ 1996; 15: 359-368.

5. Blumenauer B, Judd M, Cranney A, et al. Etanercept for the treatment of rheumatoid arthritis. Cochrane Database Syst Rev 2003; 4: CD004525.

6. Navarro-Sarabia F, Ariza-Ariza R, Hernandez-Cruz B, Villanueva I. Adalimumab for treating rheumatoid arthritis. J Rheumatol 2006; 33: 1075-1081.

7. Chen YF, Jobanputra P, Barton P, et al. A systematic review of the effectiveness of adalimumab, etanercept and infliximab for the treatment of rheumatoid arthritis in adults and an economic evaluation of their cost-effectiveness. Health Technol Assess 2006; 10: 1-229.

8. Maini R, St Clair EW, Breedveld F, et al. Infliximab (chimeric anti-tumour necrosis factor- $\alpha$ monoclonal antibody) versus placebo in rheumatoid arthritis patients receiving concomitant methotrexate: a randomised phase III trial. Lancet 1999; 354: 1932-1939.

9. Allaart CF, Lems WF, Huizinga TW. The BeSt way of withdrawing biologic agents. Clin Exp Rheumatol 2013; 31 (Suppl 78): S14-18.

10. Weinblatt ME, Keystone EC, Furst DE, et al. Adalimumab, a fully human anti-tumor necrosis factor- $\alpha$ monoclonal antibody, for the treatment of rheumatoid arthritis in patients taking concomitant methotrexate: the ARMADA trial. Arthritis Rheum 2003; 48: 35-45.

11. Moreland LW, Schiff MH, Baumgartner SW, et al. Etanercept therapy in rheumatoid arthritis. A randomized, controlled trail. Ann Intern Med 1999; 130: 478-486.
12. Wiens A, Venson R, Cassyano J, et al. Meta-analysis of the Efficacy and Safety of Adalimumab, Etanercept, and Infliximab for the Treatment of Rheumatoid Arthritis. Pharmacotherapy 2010; 30: 339-353.

13. Schabert VF, Bruce B, Ferrufino CF, et al. Disability outcomes and dose escalation with etanercept, adalimumab, and infliximab in rheumatoid arthritis patients: a US-based retrospective comparative effectiveness study. Curr Med Res Opin 2012; 28: 569-580.

14. Nagasawa H, Kameda H, Sekiguchi N, et al. Improvement of the HAQ score by infliximab treatment in patients with RA: its association with disease activity and joint destruction. Mod Rheumatol 2009; 19: 166-172.

15. Malottki K, Barton P, Tsourapas A, et. al. Adalimumab, etanercept, infliximab, rituximab and abatacept for the treatment of rheumatoid arthritis after the failure of a TNF inhibitor: a systematic review and economic evaluation. Health Technol Assess 2011; 15: 1-278.

16. Kaczor MP, Wójcik R. An economic analysis of TNF- $\alpha$ antagonists for rheumatoid arthritis in Polish settings. Reumatologia 2007; 45: 268-275.

17. Wailoo AJ, Bansback N, Brennan A, et al. Biologic Drugs for Rheumatoid Arthritis in the Medicare Program. Arthritis Rheum 2008, 58: 939-946.

18. Hidalgo-Vega A, Villoro R, Blasco JA, et al. Cost-utility analysis of certolizumab pegol versus alternative tumour necrosis factor inhibitors available for the treatment of moderate-to-severe active rheumatoid arthritis in Spain. Cost Eff Resour Alloc 2015; 13: 11.

19. Savova A, Marinov L, Georgieva S, et. al. Pharmacoeconomic assessment of biosimilar infliximab for the therapy of rheumatoid diseases in the Bulgarian health care setting - a Markov model. World J Pharm Pharm Sci 2014; 3: 147-153. 